Nephrologe 2014 · 9:425-426

DOI 10.1007/s11560-014-0866-0

Online publiziert: 12. November 2014

(c) Springer-Verlag Berlin Heidelberg 2014
F. Keller ${ }^{1}$ J.T. Kielstein ${ }^{2} \cdot$ G. Mayer ${ }^{3}$

${ }^{1}$ Abteilung Innere Medizin I, Sektion Nephrologie, Universitätsklinikum UIm

${ }^{2}$ Klinik für Nieren- und Hochdruckerkrankungen, Medizinische Hochschule Hannover

${ }^{3}$ Universitätsklinik für Innere Medizin IV, Nephrologie und Hypertensiologie,

Medizinische Universität Innsbruck, Österreich

\title{
Auswahl und Dosierung von Medikamenten bei chronischen Nierenerkrankungen
}

\author{
Ungenau, unbekannt, unbeliebt?
}

Weitere Infos auf springermedizin.de

\section{Dossier} Nephrologie

Niereninsuffizienz, Hypertonie, Elektrolyte, Transplantation: In dieser ständig aktualisierten Themensammlung dreht sich alles um die Niere.

- www.springermedizin.de/ innere-nephrologie
Patienten mit chronischen Nierenerkrankungen sind meist multimorbid. Der Diabetes mellitus oder die Hypertonie als häufige Grunderkrankungen, kardiovaskuläre Komorbiditäten, eine hohe Infektanfälligkeit und natürlich auch psychiatrische Probleme, die sich aus der Verarbeitung einer schweren, chronischen Erkrankung ergeben, stellen nicht nur diagnostisch, sondern auch therapeutisch extreme Herausforderungen dar. Die hinzukommende „Wir-setzen-nur-an-und-nie-ab“-Einstellung multipler Verordner von Medikamenten führt über Jahre häufig zu einer Polypharmakotherapie. In einer Studie aus den USA (die man aber durchaus auch auf Europa übertragen kann) lag der Median der Medikamente, die täglich von Patienten an der Hämodialyse eingenommen wurden, bei 19; 25\% der Population erhielten sogar über 25 Präparate. Neben negativen Einflüssen auf die Adhärenz weisen rezente Querschnittsuntersuchungen auch darauf hin, dass es bei 75\% der Patienten durch diese umfangreiche Therapie $\mathrm{zu}$ Medikamenteninteraktionen kommt, die bei 15-20\% ein sofortiges Einschreiten notwendig machen würden. Hier wären computergestützte Programme, die Interaktionen erkennen, ebenso wie eine verstärkte Kooperation zwischen Pharmazeuten, klinischen Pharmakologen und Klinikern theoretisch hilfreich; diese Optionen werden aber praktisch noch zu selten genutzt.

Das Hauptproblem bei der Pharmakotherapie von chronisch Nierenkranken ist, dass viele Medikamente, zumindest teil- weise, renal ausgeschieden werden. Ihre Pharmakokinetik, aber auch Pharmakodynamik verändert sich daher im Laufe der Progression der Nierenfunktionseinschränkung deutlich. Komplizierend kommt dazu, dass eine chronische Nephropathie auch die extrarenale Clearance verändern kann und eine Dosierung nach der glomerulären Filtrationsrate nicht berücksichtigt, dass Veränderungen tubulärer Exkretionsfunktionen nicht immer parallel zu jenen der glomerulären verlaufen.

\section{》) Hauptproblem bei der Pharmakotherapie chronisch Nierenkranker ist, dass viele Medikamente renal ausgeschieden werden}

Die Gruppe der Patienten mit einer weit fortgeschrittenen Nierenerkrankung ist klein, das Interesse an exakten pharmakologischen Studien in dieser Population ist gering, und die Patienten werden auch häufig aus Zulassungsstudien ausgeschlossen. Sogar bei extrakorporalen Therapieverfahren ist es nicht einfach, methodisch einwandfreie Studien zur Elimination von Medikamenten durchzuführen. So wurde lange Zeit die Clearance von Substanzen berechnet, indem man im Blutzustrom und im Dialysatabstrom die Konzentration gemessen hat. Dieses Verfahren berücksichtigt nicht, dass eine Bindung von Medikamenten an großflächige 
Dialysatoren ebenfalls massiv in die Pharmakokinetik eingreifen kann.

Jedem Arzt ist wahrscheinlich klar, dass die Beschäftigung mit der Pharmakologie bei Patienten mit Nierenerkrankungen notwendig ist, aber auch eine extreme Herausforderung darstellt, weil teilweise die Qualität der Daten, die uns zur Verfügung stehen, mangelhaft ist. Dieses Heft soll dazu beitragen, zumindest für bestimmte Substanzklassen mehr Klarheit $\mathrm{zu}$ schaffen und einige wichtige Prinzipien zu beleuchten. Je geringer die therapeutische Breite einer Substanz, desto wichtiger ist es, wenn möglich auf Spiegelbestimmungen zurückzugreifen. Darüber hinaus ist gerade auf dem Gebiet der Antibiotikatherapie auch zu beachten, dass natürlich Überdosierungen bei chronischer Niereninsuffizienz vorkommen können, allerdings Unterdosierungen zu einer beträchtlichen Gefahr werden, wenn damit Infektionen nicht adäquat therapiert werden und/oder Keimresistenzen entstehen. Insbesondere bei Patienten mit chronischer Nierenerkrankung gilt es, in regelmäßigen Abständen die Indikation und das aktuelle Nutzen-Risiko-Verhältnis jedes einzelnen Medikaments zu überprüfen, denn, wie Antoine de Saint-Exupéry einst geschrieben hat, erreicht man Perfektion nicht, wenn nichts mehr hinzuzufügen ist, sondern dann, wenn nichts mehr weggelassen werden kann.

Die Herausgeber

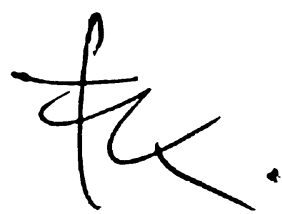

Frieder Keller

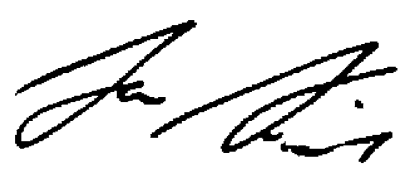

Jan T. Kielstein

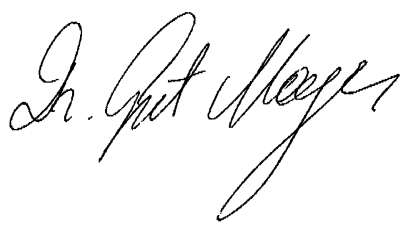

Gert Mayer

\section{Korrespondenzadressen

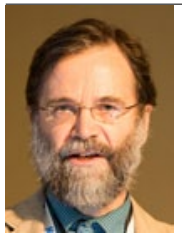 \\ Prof. Dr. F. Keller \\ Abteilung Innere Medizin I, \\ Sektion Nephrologie, \\ Universitätsklinikum Ulm \\ Albert-Einstein-Allee 23, \\ 89081 Ulm \\ frieder.keller@uniklinik-ulm.de}

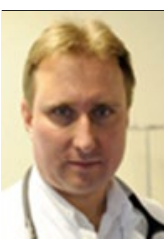

Prof. Dr. J.T. Kielstein

Klinik für Nieren- und

Hochdruckerkrankungen,

Medizinische Hochschule

Hannover

Carl-Neuberg-Str. 1,

30625 Hannover

kielstein@yahoo.com

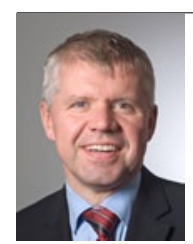

Dr. G. Mayer

Universitätsklinik für Innere

Medizin IV, Nephrologie und

Hypertensiologie,

Medizinische Universität

Innsbruck

Anichstraße 35,

A-6020 Innsbruck, Österreich gert.mayer@i-med.ac.at

Interessenkonflikt. F. Keller hat Honorare und Fortbildungsunterstützung von den Firmen Novartis, Alexion, Astellas, Roche und Amgen erhalten.

J.T. Kielstein und G. Mayer geben an, dass kein Interessenkonflikt besteht.

\section{Kleinräumige Versorgungs- forschung}

Gesundheitspolitik, die Krankenkassen und ihre Vertragspartner sowie Versicherte und Patienten stehen vor der Herausforderung, unser Gesundheitssystem an sich verändernde strukturelle und finanzielle Rahmenbedingungen anzupassen. Der kleinräumigen Versorgungsforschung

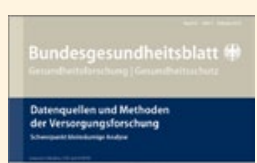
wird die Aufgabe zuwachsen, Politik, Vertragspartner und Öffentlichkeit darüber zu

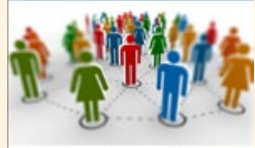
informieren, $o b$ und ggf. wie eine größtmögliche Gleichwertigkeit

der Lebensverhältnisse in der medizinischen Versorgung gewahrt bzw. wiederhergestellt werden kann.

Die Ausgabe 2/2014 der Zeitschrift Bundesgesundheitsblatt erläutert die spezifischen Fragestellungen, Methoden und Verbindungen zwischen Wissenschaft und Praxis im Versorgungsgeschehen in unter anderem folgenden Beiträgen:

- John E. Wennberg, Pionier der regionalen Versorgungsforschung

- Versorgungsforschung aus Sicht der Bundesärztekammer unter Berücksichtigung kleinräumiger Analysen

- Statistische Methoden der kleinräumigen Versorgungsforschung

- Datenlinkage von Primär- und Sekundärdaten

- Lernen aus regionalen Unterschieden

Bestellen Sie diese Ausgabe zum Preis von 16,- EUR zzgl. Versandkosten bei Springer Customer Service Center Kundenservice Zeitschriften Haberstr. 7, 69126 Heidelberg Tel. +49 6221-345-4303

Fax: +49 6221-345-4229

E-Mail: leserservice@springer.com

Suchen Sie noch mehr zum Thema? Mit e.Med, dem Online-Paket von Springer Medizin, können Sie schnell und komfortabel in über 600 medizinischen Fachzeitschriften recherchieren.

Weitere Infos unter springermedizin.de/ eMed. 